in vivo $34: 1951-1963(2020)$

doi:10.21873/invivo.11992

\title{
Endometrial Dating Method Detects Individual Maturation Sequences During the Secretory Phase
}

\author{
JOACHIM ALFER ${ }^{1,2}$, AMIR FATTAHI $^{3,4}$, NATHALIE BLEISINGER ${ }^{3}$, JÜRGEN KRIEG ${ }^{5}$, \\ ROLF BEHRENS $^{6}$, RALF DITTRICH ${ }^{3}$, MATTHIAS W. BECKMANN ${ }^{3}$, ARNDT HARTMANN ${ }^{1}$, \\ IRMGARD CLASSEN-LINKE ${ }^{7}$ and ROXANA M. POPOVICI ${ }^{8}$ \\ ${ }^{1}$ Department of Pathology, Erlangen University Hospital, \\ Friedrich-Alexander University of Erlangen-Nürnberg, Erlangen, Germany; \\ ${ }^{2}$ Kaufbeuren-Ravensburg Institute of Pathology, Ravensburg, Germany; \\ ${ }^{3}$ Department of Obstetrics and Gynecology, Erlangen University Hospital, \\ Friedrich-Alexander University of Erlangen-Nürnberg, Erlangen, Germany; \\ ${ }^{4}$ Department of Reproductive Biology, School of Advanced Medical Sciences, \\ Tabriz University of Medical Sciences, Tabriz, Iran; \\ ${ }^{5}$ Fertility-Center Amberg, Amberg, Germany; \\ ${ }^{6}$ Centre for Reproductive Medicine, Erlangen, Germany; \\ ${ }^{7}$ Institute of Molecular and Cellular Anatomy, Medical Faculty, RWTH Aachen University, Aachen, Germany; \\ ${ }^{8}$ Fertility-Center Munich, Munich, Germany
}

\begin{abstract}
Background/Aim: This study assessed whether a new immunohistochemical dating method allows precise endometrial dating allowing optimal timing for embryo transfer. Patients and Methods: A novel method was used for endometrial dating, with parameters including menstrual cycle days, Noyes histological criteria, along with immunohistochemical expression pattern of estrogen and progesterone receptors and proliferation marker Ki-67. Endometrial maturation was analyzed on days +5 to +10 after ovulation or progesterone administration in 217 biopsies from 151 subfertile patients during the secretory phase. Results: Endometrial maturation varied individually, occurring 1.68 \pm 1.67 days late. Comparison of histological maturation with clinical days after ovulation showed a delay of about 2 days. Conclusion: Endometrial maturation requires 8 days, rather than the expected 6 days, to reach the histological mid-secretory phase. This is not a delay and is also seen in fertile patients. The new analysis method used
\end{abstract}

This article is freely accessible online.

Correspondence to: Joachim Alfer, MD, Institut für Pathologie Kaufbeuren-Ravensburg, Elisabethenstrasse 19, 88212 Ravensburg, Germany. Tel: +49 75118086740, Fax: +49 75118086769, e-mail: joachim.alfer@pathologie-ravensburg.de

Key Words: Endometrial dating, estrogen receptor, progesterone receptor, endometrial receptivity, window of implantation. is superior to that using Noyes criteria alone and provides a better basis for determining conditions for optimal timing of embryo transfers.

The endometrium is one of the major factors involved in embryo implantation. However, the process involved and the underlying molecular mechanisms that enable the endometrium to enter the receptive phase are still not fully clear. Many researchers have explored various methods for investigating endometrial maturation during the menstrual cycle. Well-dated endometrial tissue is required in order to study the molecular features of the endometrium during the menstrual cycle, and inadequate dating can lead to misinterpretation even if the structure of a research study is excellent. In order to identify the receptive phase in the endometrium, especially in patients with suspected endometrial factor infertility, endometrial biopsies need to be taken with precise timing. Exact maturation sequencing of the endometrium is also required.

Molecular methods simultaneously analyzing hundreds of genes appeared likely at one time to become the new standard, offering new ways of predicting the implantation window (1). In addition to the high cost of the endometrial receptivity array and its complexity, however, further studies showed that performing this test in a mock cycle before an embryo transfer did not improve the ongoing pregnancy rate in patients who had a good prognosis $(2,3)$.

Considerable efforts have been made to develop an alternative to the classical histomorphological dating of the 
endometrium. In 1988, $\mathrm{Li}$ and colleagues described 17 morphometric measurements, only five of which showed a highly significant correlation with chronological dating based on a surge in luteinizing hormone (4). One of the important studies in this field was conducted by Noyes et al., who carried out a chronological analysis of histological changes in endometrial biopsies for each day during the menstrual cycles of subfertile patients (5). In particular, they evaluated gland mitoses, pseudostratification of nuclei, basal vacuolation, secretion, stromal edema, pseudodecidual reaction, stromal mitoses, and leukocytic infiltration. Many researchers have used Noyes criteria to analyze the quality of endometrial dating, and have reported a wide range of intraobserver variance, which may have a significant impact on clinical management (6). In a study including 176 infertile women, dating of endometrial biopsies showed that only $64 \%$ of patients were in the secretory phase, while the rest $(36 \%)$ had a secretory delay of at least 2 days (7). Interestingly, a wide range of individual endometrial maturation was observed in a study including fertile women (8). In a similar study including 33 healthy women with regular menstrual cycles, one-third of the endometrial biopsies were out of phase, clearly indicating the variable duration of the secretory phase among women (9). A delayed endometrium was also detected in $47 \%$ of infertile patients and $24 \%$ of fertile patients in a study conducted by Lindhard et al. (10). Coutifaris et al. (11) also reported that histological dating of endometrial biopsies from fertile and infertile patients showed out-of-phase endometrium in the mid-luteal and late luteal phases in both the fertile and infertile groups. The authors concluded that histological dating did not distinguish between fertile and infertile women.

Murray et al. investigated the accuracy, reproducibility, and clinical utility of histological endometrial dating in fertile women and reported that endometrial histological dating criteria are not sufficiently accurate to provide a valid method for diagnosing luteal phase deficiency or guiding clinical management in women with reproductive failure. They also concluded that neither traditional dating criteria nor any combination of the best histological features was reliably able to distinguish any specific day in the cycle or narrow interval of days (12).

In a previous study, Classen-Linke et al. investigated endometrial biopsies from carefully examined fertile patients (13). Dating was carried out using Noyes criteria in combination with clinical data, immunohistochemical analyses of hormone receptors and $\mathrm{Ki}-67$, and also serum hormone analyses of luteinizing hormone, follicle-stimulating hormone, estrogen, and progesterone. It was found that progesterone receptor (PR) and estrogen receptor (ER), as well as proliferation marker Ki-67, showed a dynamic expression pattern during the early, mid-luteal, and late luteal phases (13).
The aim of the present study was to combine the histomorphological criteria of Noyes et al. (5), which were established for endometrial biopsies from patients with sterility, with immunohistochemical analyses of hormone receptors for PR and ER, as well as Ki-67, on a day-by-day basis in order to obtain more precise dating results.

\section{Patients and Methods}

Study population. This study recruited 151 patients with subfertility (mean age $=37.19 \pm 4.16$ years) who had at least one good embryo based on the Cutting et al. criteria (14). The patients had experienced implantation failures after in vitro fertilization a median of four times. The patients were divided into two groups. In group A $(\mathrm{n}=128), 172$ biopsies were obtained in well-monitored natural cycles with natural ovulation after the luteinizing hormone peak, while in group B, 45 biopsies were taken in 23 patients in a mock hormone replacement therapy (HRT) cycle.

In group A, one biopsy was taken in one menstrual cycle from 84 patients, one biopsy was taken in two different cycles from eight patients, and two biopsies were taken between 5 and 10 days after ovulation (OV) from 36 patients, so that a total of 172 biopsies were investigated.

In group B, one biopsy was taken in one menstrual cycle from one patient, two biopsies were taken in two different cycles from four patients, and two biopsies were taken in one menstrual cycle from 18 patients, so that a total of 45 biopsies were taken. Biopsies were taken after 5 days and after 10 days of progesterone administration.

HRT was administered on the basis of the standard Kaufmann protocol (15), including estrogen and progesterone (P) administration, with the latter starting on day 12 of estrogen application. In all patients, the biopsies were obtained using a Pipelle endometrial suction curette (Gynemed GmbH, Lensahn, Germany) between OV/P +5 and +10 days, corresponding to days 19 and 24 of the menstrual cycle. Patients who were found on sonohysterography to have an abnormal uterine cavity, unilateral or bilateral hydrosalpinx, or endometrium $\leq 6 \mathrm{~mm}$ were excluded from the study. The patients' clinical examinations did not reveal any pathological findings, with adequate ovulation, optimal hormone levels in peripheral blood, and typical development of the endometrium with a triple-line pattern, as detected on ultrasound.

The study was approved (approval number: 322_19 Bc) by the Ethics Committee of the Medical Faculty of Friedrich-Alexander University of Erlangen-Nürnberg.

Immunohistochemistry of endometrial markers. For immunostaining, paraffin-embedded tissue sections were cut $(1 \mu \mathrm{m})$ and fixed on slides; rehydration was achieved using an ethanolic series. Briefly, staining was carried out by incubating the sections with diluted primary monoclonal antibodies for PR, ER, and Ki-67 (1:300, 1:400, and 1:500, respectively) for $45 \mathrm{~min}$ at room temperature. The primary antibodies for PR and ER were obtained from DCS Innovative Diagnostic Systems (Hamburg, Germany) and antibodies for Ki-67 (MIB-1) from Zytomed Systems (Berlin, Germany). After the slides had been washed in washing buffer (Zytomed) and incubated with PostBlock reagent (POLAP-100-Kit; Zytomed), they were incubated with AP polymer (POLAP-100-Kit; Zytomed) for 30 min at room temperature. The slides were then washed and stained 
Table I. Dating method using immunohistochemical positivity.

\begin{tabular}{lccccc}
\hline & \multicolumn{2}{c}{ Glands } & & \multicolumn{2}{c}{ Ki-67 (\%) } \\
\cline { 2 - 3 } \cline { 5 - 6 } Day of cycle & ER $(\%)$ & PR (\%) & & Glands & Stroma \\
\hline 16 & $>80$ & $>80$ & & $>15$ & $<8$ \\
17 & $50-90$ & $85-95$ & & $<10$ & $<10$ \\
18 & $20-60$ & $55-85$ & & $<2$ & $<10$ \\
19 & $10-30$ & $15-55$ & & $<1$ & $<15$ \\
20 & $<20$ & $<20$ & & $<1$ & $<15$ \\
21 & $<10$ & $<2$ & & $<1$ & $10-20$ \\
22 & $<10$ & 0 & & 0 & $15-25$ \\
23 & $<5$ & 0 & & 0 & $20-25$ \\
24 & $<3$ & 0 & & 0 & $25-35$ \\
\hline
\end{tabular}

ER: Estrogen receptor; PR: progesterone receptor.

with chromogen solution (Permanent Red AP-Kit; Zytomed) for 20 min at room temperature. After the final washing and dehydration, the coverslip was mounted and the slides were evaluated under a microscope. To confirm the immunostaining method, positive control tissues were attached to each slide for Ki-67 (appendix cross-section) and a cell control array was used for hormone receptors (Zytomed). Receptor analysis of the glands was carried out in the stratum functionale of the endometrium, and the complete biopsy was analyzed and classified at 5\% interval steps. When necessary, counting was also carried out, especially when fewer than $5 \%$ of stained nuclei were visible. For Ki-67 analysis, positively stained nuclei of glandular epithelium in the stratum functionale of the endometrium were evaluated. Three hundred stromal cells were analyzed by counting three representative fields.

Endometrial dating method. The endometrial biopsies were analyzed in accordance with previously published criteria $(13,16)$ during natural cycles $(\mathrm{OV}+5$ to $\mathrm{OV}+10)$ as well as HRT cycles $(\mathrm{P}+5$ and $\mathrm{P}+10$ ) and were dated on the basis of a novel method. Various parameters were taken into account for endometrial dating, including menstrual cycle days and histological dating criteria based on the Noyes protocol (5) for all biopsies, in combination with the immunohistochemical expression pattern of ER and PR and proliferation marker Ki-67 in 184 biopsies. This method assigned a pattern of related receptor and Ki-67 expression to each histomorphological appearance between cycle days 16 and 24 due to the endometrial maturation delay of 1-3 days from biopsies taken at day 19-24.

The combined dating criteria for cycle days 16-24 are listed in Table I (dating method).

\section{Results}

Modified dating method. Analysis of the biopsies taken at $\mathrm{OV} / \mathrm{P}+5$ to $\mathrm{OV} / \mathrm{P}+10$ using the modified dating method showed results representing a wide range of histomorphological patterns corresponding to cycle days 1624. Figure 1 shows the histomorphological findings for each cycle day between days 16 and 24 based on this method. Figure 2 shows the corresponding expression patterns of ER and PR, as well as Ki-67, for the histological cycle days between 16 and 24 . The histomorphology of the initial early secretory endometrium was regarded as representing the early stage of the secretory phase, corresponding to day 16 based on the new dating method, which showed proliferating glands and high levels of hormone receptor expression in the glands. The typical histomorphology of cycle day 17, with large retronuclear glycogen vacuoles, showed a slight reduction in the hormone receptors, with a proliferation index below 5\% in the glands. Receptor expression exceeding $50 \%$ in the glands, with only scattered proliferating epithelial cells and an increasing proliferation index in the stroma, was observed on histomorphology of day 18. On histomorphology of day 19, receptor expression was below $50 \%$ in the glands and the proliferation index increased in the stroma. This method, which is based on the expression pattern in combination with histomorphological analysis, was thus able to distinguish clearly between the start of the early secretory phase and the end of the early secretory phase, which represents the beginning of the window of implantation. Histomorphology of cycle days 2024 showed predominantly stromal changes, with the start of a pre-decidual reaction, spiral artery growth, and increasing stromal proliferation.

In some cases, the dating results were in between two histomorphology of cycle days, for example, day 18.5 of a cycle, because the features and receptor expression of two different cycle days were evident. It was therefore decided to take continuous histological changes into account by assigning half a day in between.

The results show that protein levels of ER and PR in the endometrial glandular epithelium significantly declined from $\mathrm{OV} / \mathrm{P}+5$ to $\mathrm{OV} / \mathrm{P}+10$ in both natural and HRT cycles (Figure $3 \mathrm{~A}$ and $\mathrm{B})$. In the natural cycle, estrogen and progesterone receptor levels in glandular epithelium ranged from $87 \pm 8 \%$ and $91 \pm 7 \%$ on day 16 to $40 \pm 19 \%$ and $69 \pm 14 \%$ on day 18 , and also $2 \pm 1 \%$ and $0 \pm 0 \%$ on day 24 , respectively. Similar changes were observed in the HRT cycle; however, ER and PR levels were significantly higher on days 18 and 19 in the HRT cycle in comparison with the natural cycle $(p<0.05)$. Days 20 and 21 are not shown in Figure 3B because no endometrial biopsies related to these days of the menstrual cycle were obtained, possibly due to low biopsy numbers in the HRT group.

Ki-67 levels in natural cycles declined significantly in the glandular epithelium from days 16 to $19(p<0.05)$. Ki-67 expression was not observed on days 20 to 24 , while its expression in stromal cells increased significantly from day 16 to day $24(p<0.05$; Figure $3 C)$. A similar pattern was observed in the HRT group (Figure 3D); however, the results showed that glandular epithelium from HRT cycles had lower levels of Ki-67 in comparison with the natural cycles on days 16 to $19(p<0.05)$. Stromal cells from HRT cycles 
also showed lower Ki-67 levels in comparison with natural cycles on days 17,18 , and $23(p<0.05)$.

When the endometrial dating results were compared with the test results including immunohistochemistry using only Noyes criteria, a discrepancy was found for $56.96 \%$ of the declared cycle days. A deviation of plus or minus half a day was evident in $25.31 \%$ of cases due to dating diagnoses ahead of the tests, with dating results lying between two cycle days. Deviations of plus or minus 1 day and more than 1 day were present in $15.18 \%$ and in $16.03 \%$ of the results, respectively. Deviation of 2 or more days was detected in $6.33 \%$.

Since combining Noyes criteria with the typical immunohistochemical pattern was able to classify the endometrial day of maturation more reliably, endometrial dating was carried out for 128 women with natural cycles and 23 women receiving HRT. Surprisingly, the novel dating method showed a clear and individual delay in endometrial maturation in most cases. For example, endometrial biopsies on $\mathrm{OV}+5$ (26 patients, natural cycle) showed the expected maturation state in only $12 \%$ of cases; $81 \%$ showed a delay of 0.5-3.5 days, and 7\% were at day 20 (Figure 4A). It was found that natural cycle biopsies on day OV+6 (19 patients) showed a delay in $95 \%$ of cases, with a mean dating result of $\mathrm{OV}+3.74 \pm 1.05$ days (Figure 4B). In addition, biopsies on $\mathrm{OV}+7$ (42 patients), $\mathrm{OV}+8$ (nine patients), $\mathrm{OV}+9$ (nine patients), and $\mathrm{OV}+10$ (23 patients) showed mean dating results of $\mathrm{OV}+5.27 \pm 1.31,6.72 \pm 1.13,7.5 \pm 0.92$, and $8.37 \pm 0.95$ days, respectively. The expected maturation was only seen in $9 \%, 22 \%, 10 \%$, and $12 \%$ of the cases, respectively (Figures $4 \mathrm{C}-\mathrm{F}$ ). These results demonstrate that although most biopsies on $\mathrm{OV}+8$ to $\mathrm{OV}+10$ showed some delay, they were mostly within the window of implantation (defined as days 20-24).

At day $\mathrm{P}+5$ in the HRT group, the endometrial tissues were in delay for 1 day in $33 \%$ of cases $(n=6), 2$ days in $39 \%(n=7)$, and 3 days in $17 \%(n=3)$, with only $11 \%(n=2)$ reaching the expected day (Figure 5A). Surprisingly, all of the biopsies on day $\mathrm{P}+10$ had delays, and none of them reached the expected maturation (Figure 5B). However, nearly all of them were within the window of implantation, starting at P+6 (corresponding to day 20).

Comparison of the histological maturation findings after ovulation with clinical days after ovulation showed a maturation delay of about 2 days. The early secretory phase thus required 8 days $(\mathrm{OV} / \mathrm{P}+8)$ instead of 6 days to develop into the clinically expected histomorphological day 20 and thus enter the expected window of implantation (Figure 6).

\section{Discussion}

Many studies have evaluated female fertility by analyzing endometrial specimens from subfertile patients. Most of these have reported poor reproducibility and accuracy, with high levels of intraobserver and interobserver variability (11, 12). Research has therefore been carried out to identify new, more precise markers and criteria for endometrial dating. In a study on women with confirmed fertility and normal menstruation, for example, it was suggested that the temporal and morphological characteristics of pinopods on the surface of the endometrium during the secretory phase might serve as a marker for endometrial dating (17).

The human endometrium is a complex organ with typical histological changes, which were first described by Rock and Bartlett (18), who correlated the dating of 200 endometrial biopsies with the day on which menstruation occurred. The authors found that $17 \%$ of women menstruated later than expected, $16 \%$ on the predicted day, and $68 \%$ earlier. Noyes et al. (5) later investigated the endometrium (sterility biopsies) in the secretory phase in 300 women during routine sterility studies and reported that the first day of menstruation correlated with the histologically expected day. They therefore introduced histological criteria for endometrial dating that have since become the gold standard. Noyes and colleagues demonstrated that among the 300 patients, $14 \%$ menstruated on the predicted day, $12 \%$ later than expected, and $74 \%$ earlier than expected. They observed errors ranging from 12 days early to 8 days late, giving a mean of $1.81 \pm 2.33$ days for early menstruation. Using the

Figure 1. Hematoxylin-eosin staining for histological analysis of the endometrium on days 16-24 of the menstrual cycle, with key features under Noyes criteria. Key features: Day 16: Subnuclear vacuolation of the glandular epithelium is regular and becomes prominent, pseudostratification of the nuclei is still visible in the glandular epithelium, and stroma is dense with nearly naked nuclei. Day 17: Only focal stratification of the glandular epithelium is present, nuclei are more or less in a row, with homogeneous cytoplasm above them and large vacuoles below. Day 18: There is almost no stratification of the glandular epithelium. Vacuoles have decreased in size and slip past the nucleus into the cytoplasm nearer the glandular lumen and thence into the lumen. Day 19: Few vacuoles remain. Superficially, this phase may resemble the early vacuolation of the 16-day phase. The presence of intraluminal secretion and absence of pseudostratification and of mitoses serve to characterize it. Day 20: Secretion reaches its peak. Subnuclear vacuoles are rare and the nuclei are round and basally located. Secretion, positively stained with periodic acid-Schiff reaction (red colour), becomes prominent and is visible until day 25. Start of stromal edema. Day 21: Stromal edema increases. Day 22: Stromal edema reaches its peak. The spiral arterioles are not prominent, and stromal cells are nearly naked nuclei, with only thin cytoplasm. Day 23: Spiral arterioles become prominent due to condensation of their surrounding stroma. The nuclei and the cytoplasm of the periarteriolar stromal cells become larger. This is the earliest predecidual reaction. Day 24: Spiral arterioles become prominent due to the pre-decidual reaction around them. Pre-decidual changes start to differentiate focally under the surface epithelium. This is normally expected at day 25 by Noyes criteria, but in the present study it was seen irregularly in combination with pre-decidual reactions around spiral arterioles, suggesting that it starts on day 24. Stromal proliferation resumes. Magnification: Main image $\times 100$; inset: $\times 400$. 


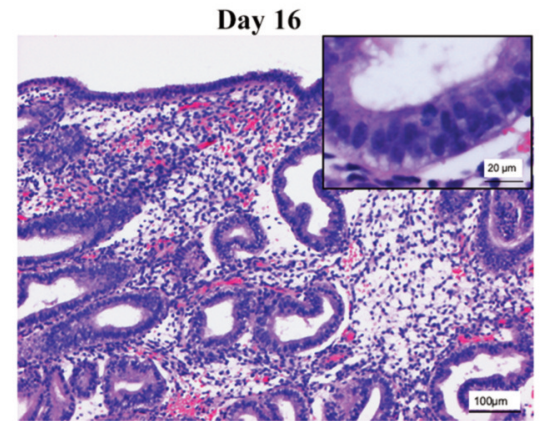

Day 18

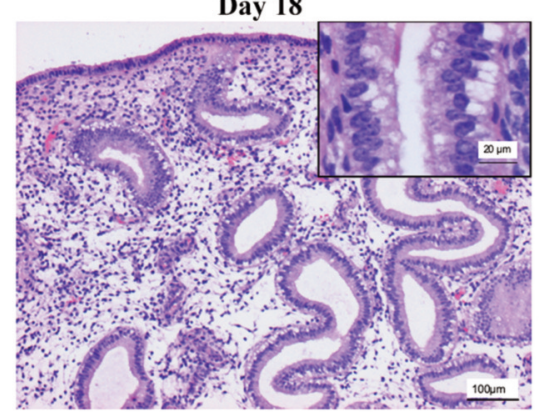

Day 20

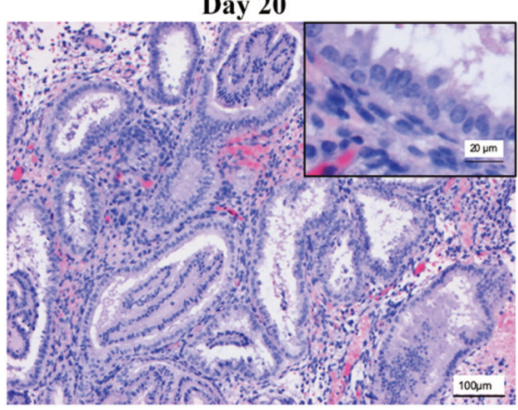

Day 22

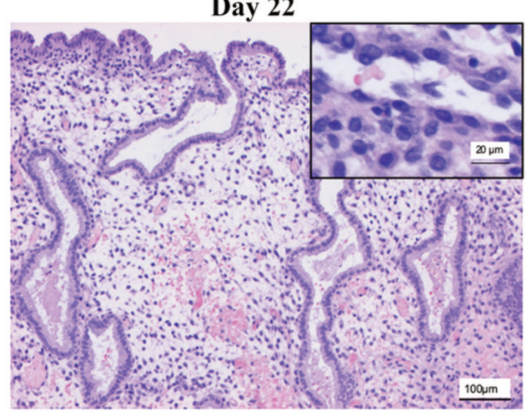

Day 17

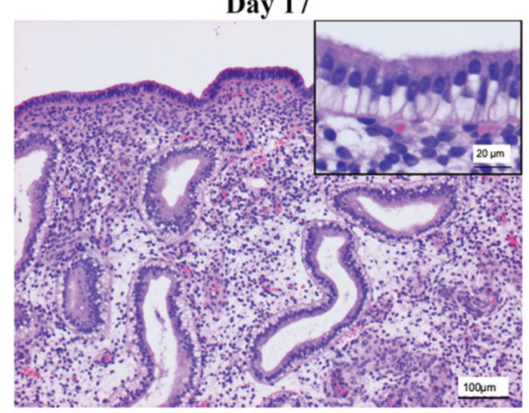

Day 19

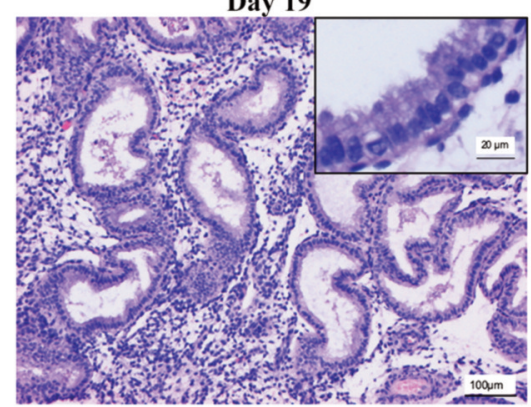

Day 21

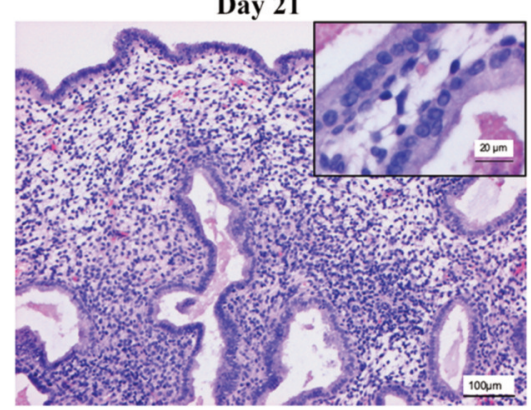

Day 23

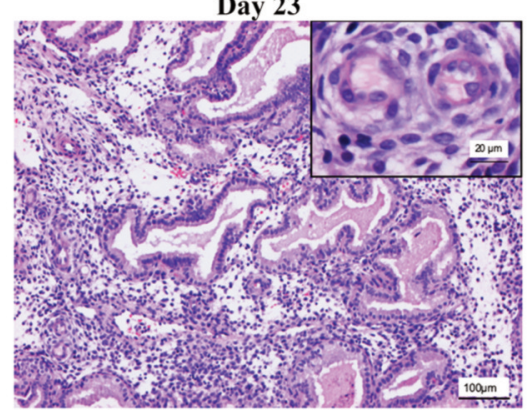

Day 24

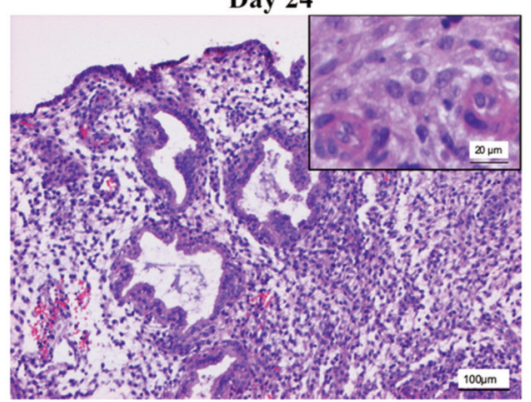


A

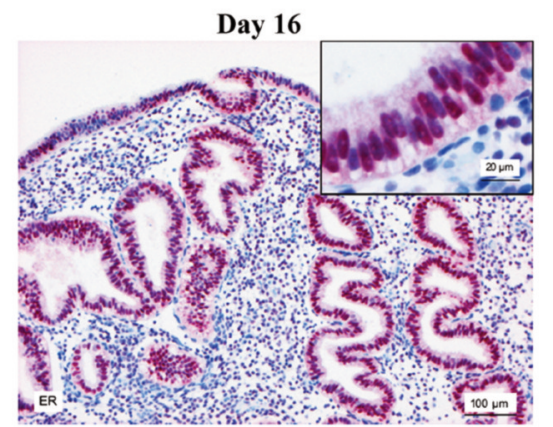

Day 18

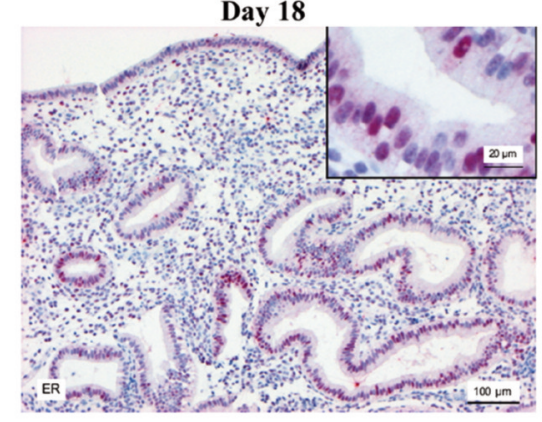

Day 20

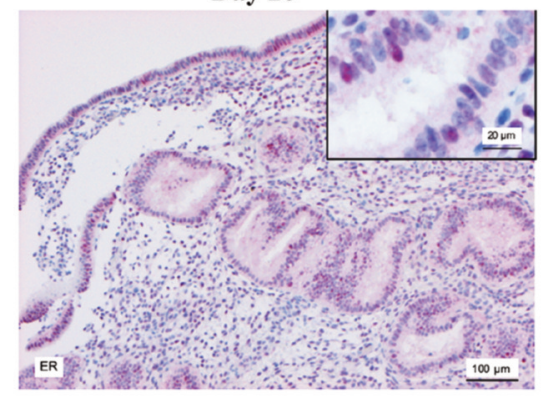

Day 22

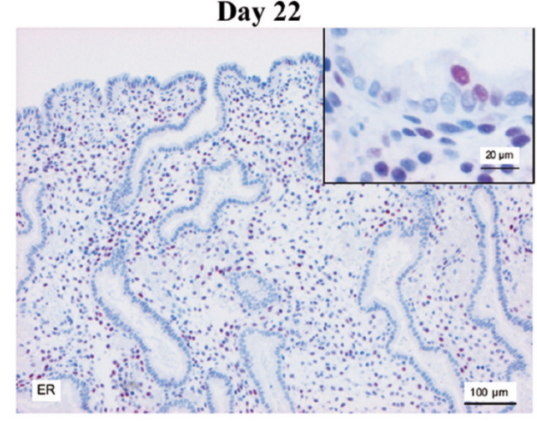

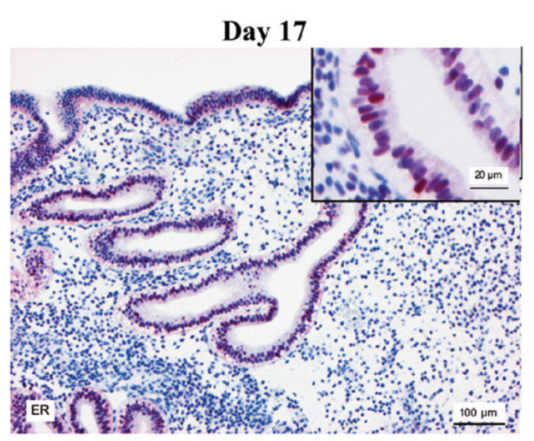

Day 19

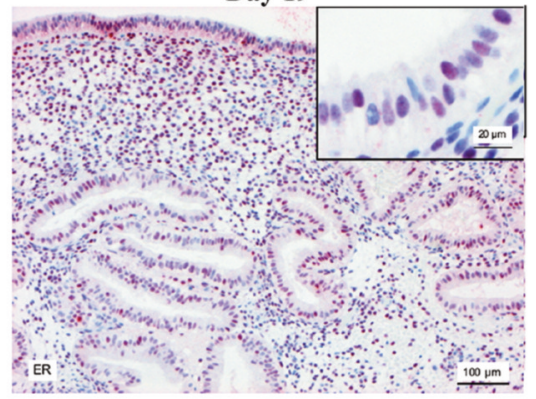

Day 21

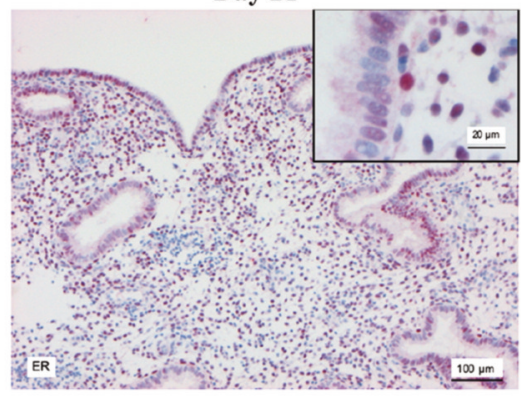

Day 23

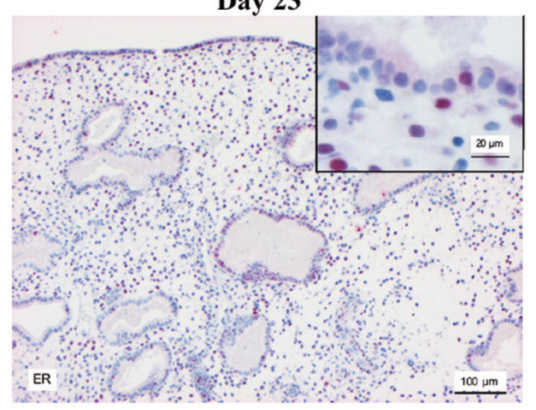

ER A

Day 24

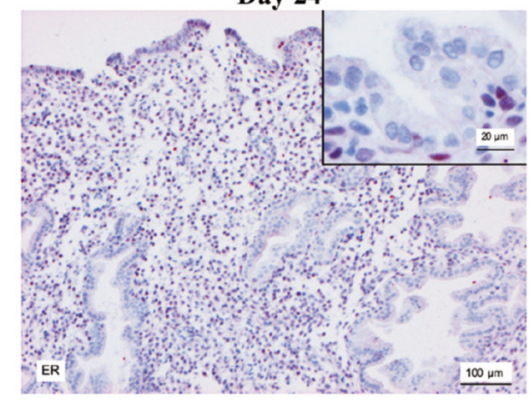

Figure 2. Continued 
B

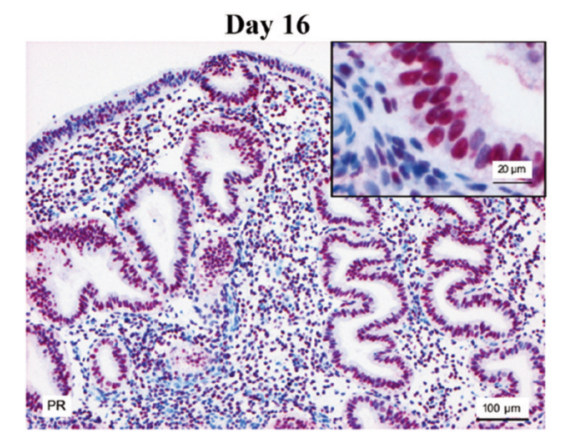

Day 18

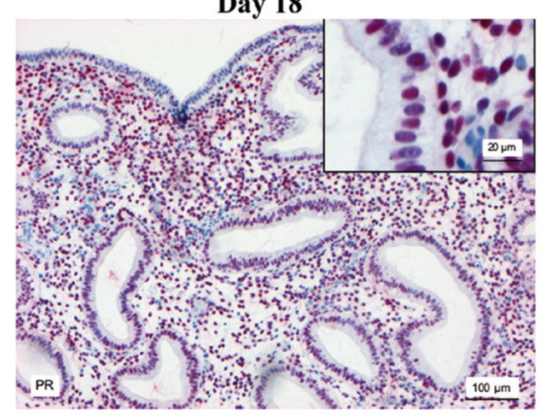

Day 20

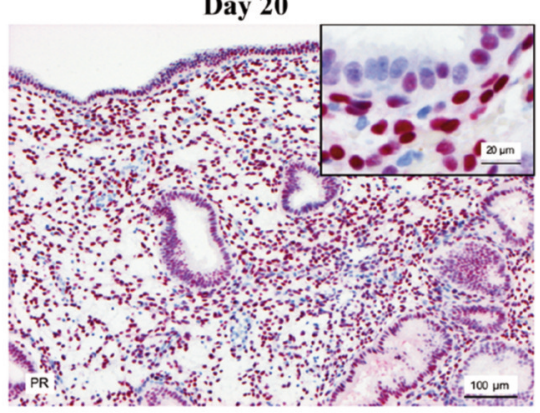

Day 22

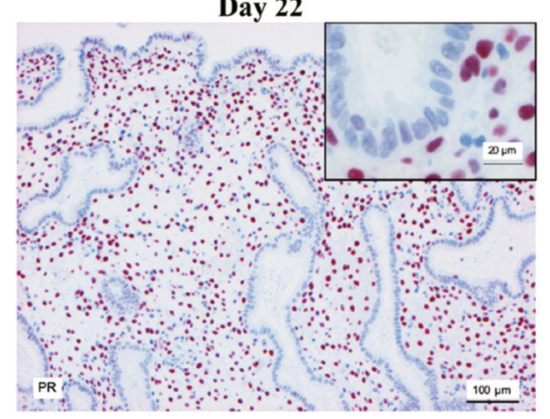

Day 17

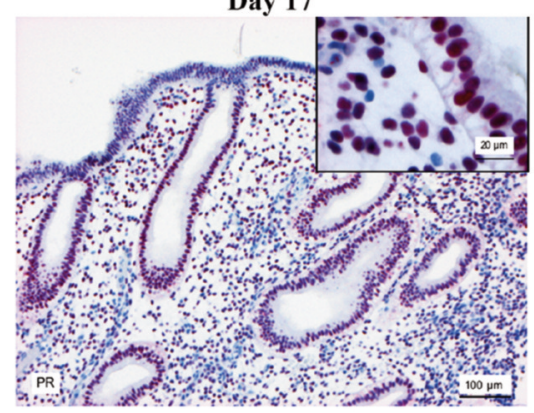

Day 19

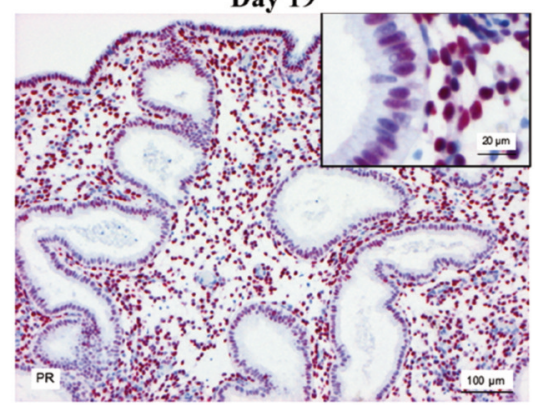

Day 21

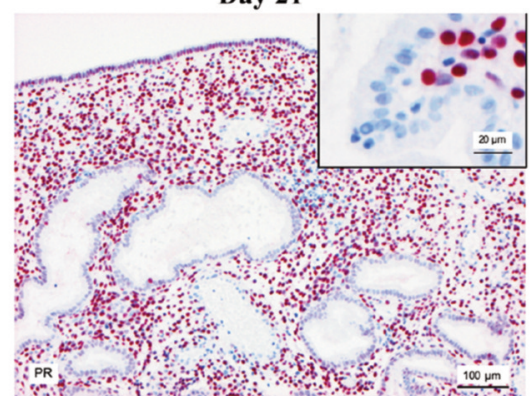

Day 23

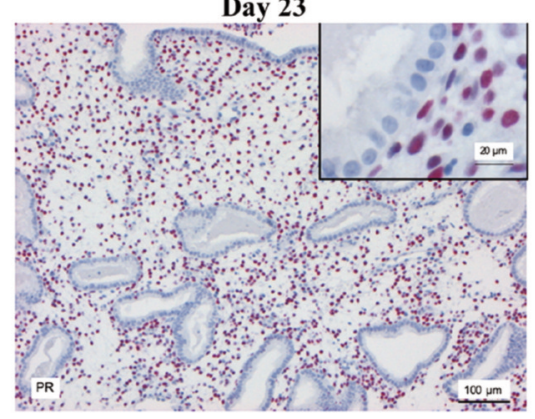

Day 24

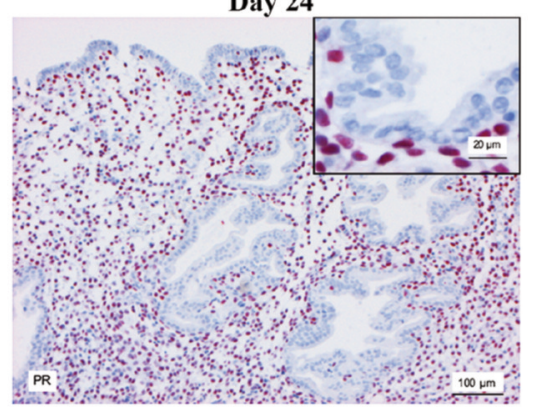

Figure 2. Continued 

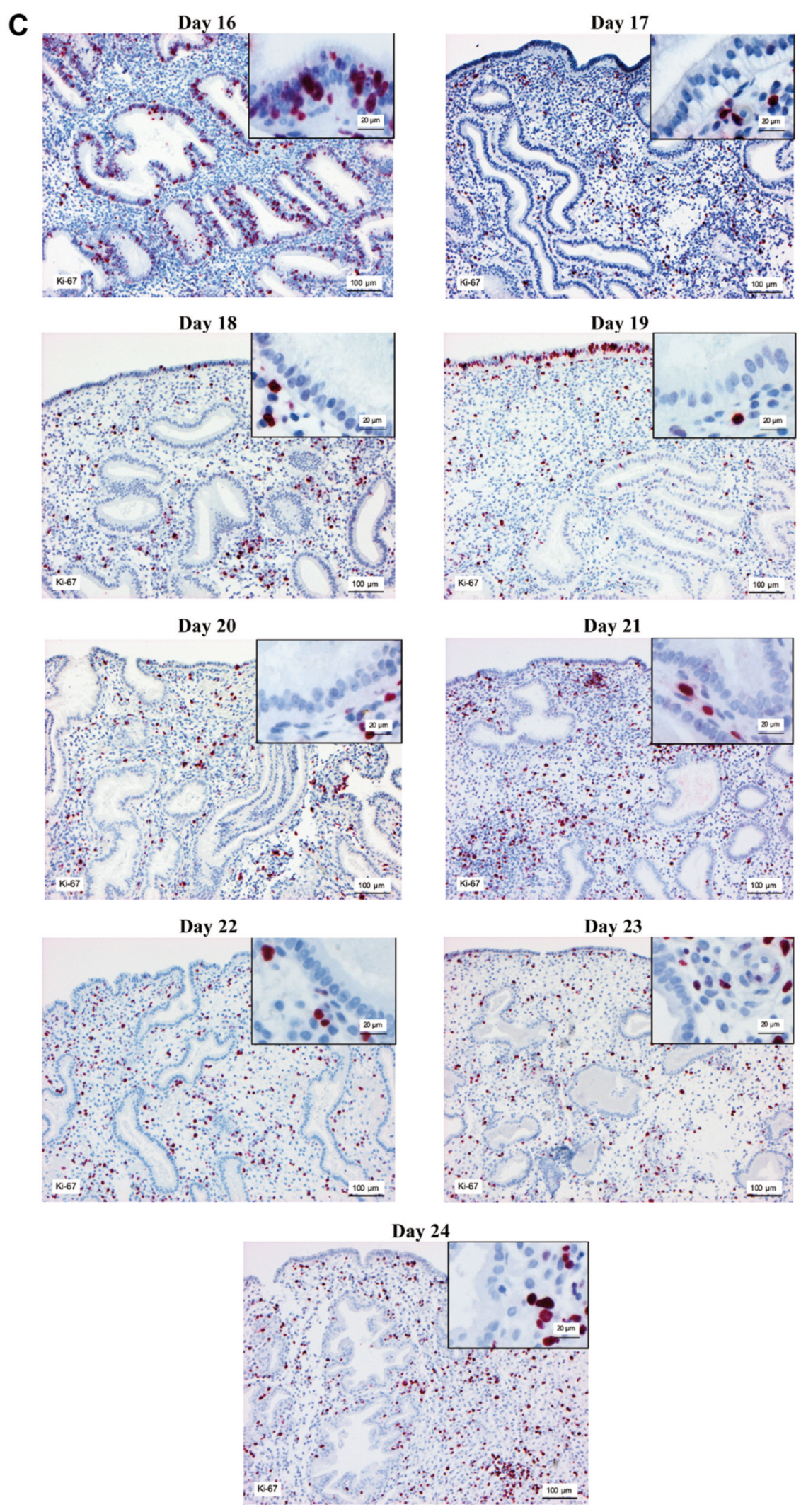

Figure 2. Immunohistochemical staining of estrogen receptor $(A)$ progesterone receptor $(B)$ and Ki-67 $(C)$ in the endometrium on days 16-24 of the menstrual cycle. Magnification, main image: $\times 100$, and inset: $\times 400$. 

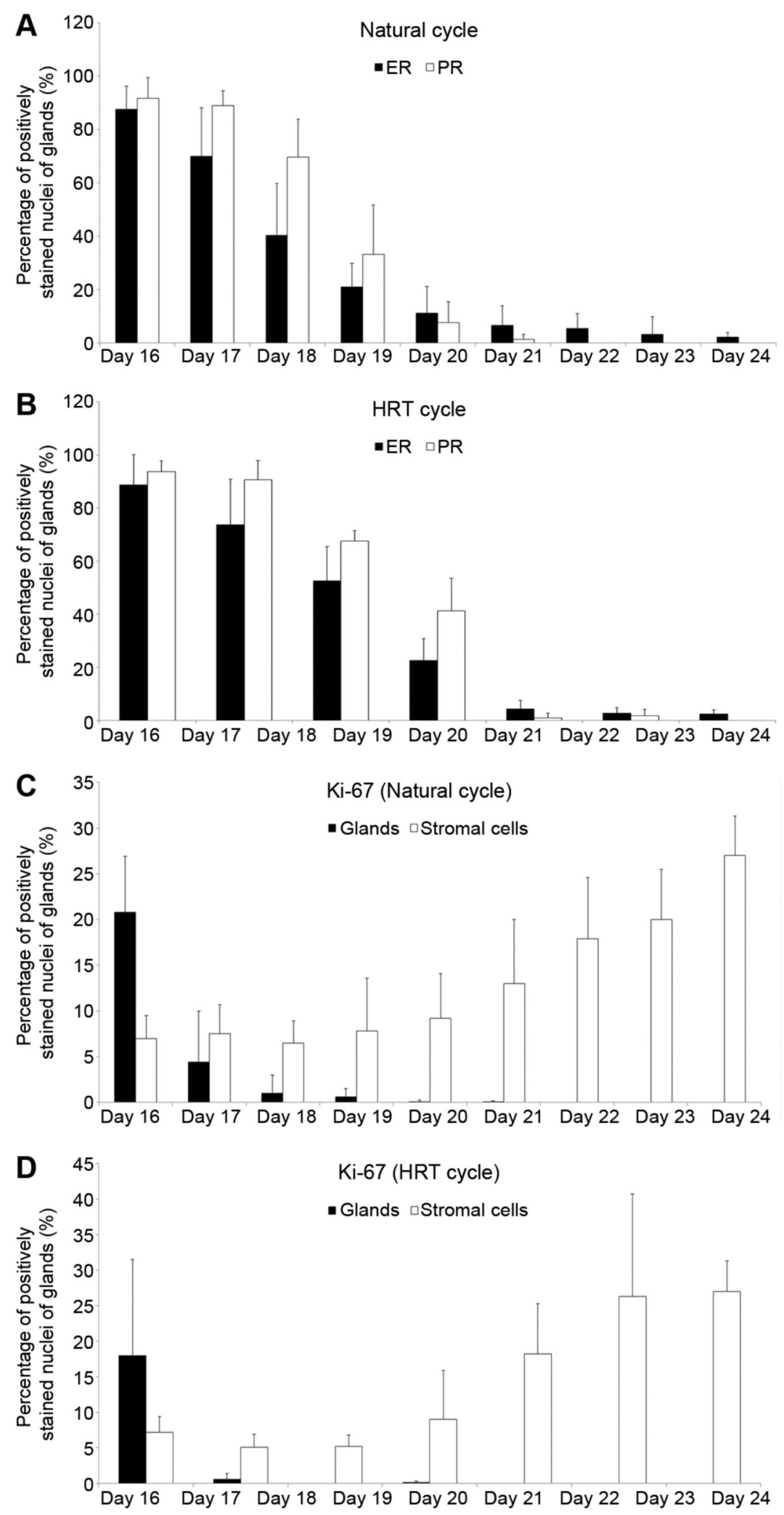

Figure 3. Percentage of immunohistochemically positively stained nuclei of endometrial glandular epithelial cells for estrogen (ER) and progesterone $(P R)$ receptors, and Ki-67 for immunohistological days 16-24 in natural cycles and hormone replacement therapy (HRT) cycles. A: ER and PR expression in natural cycles. B: ER and PR expression in HRT cycles. C: Ki-67 expression in natural cycles. (D) Ki-67 expression in HRT cycles. 
$\mathbf{A}$

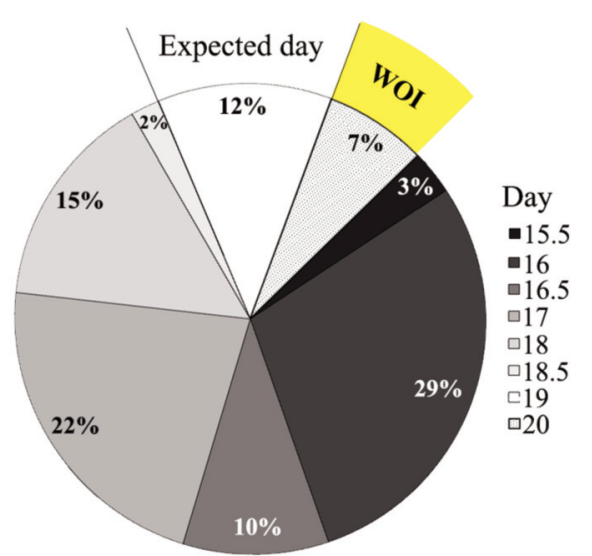

Biopsy at OV+5 (Natural cycle)

C

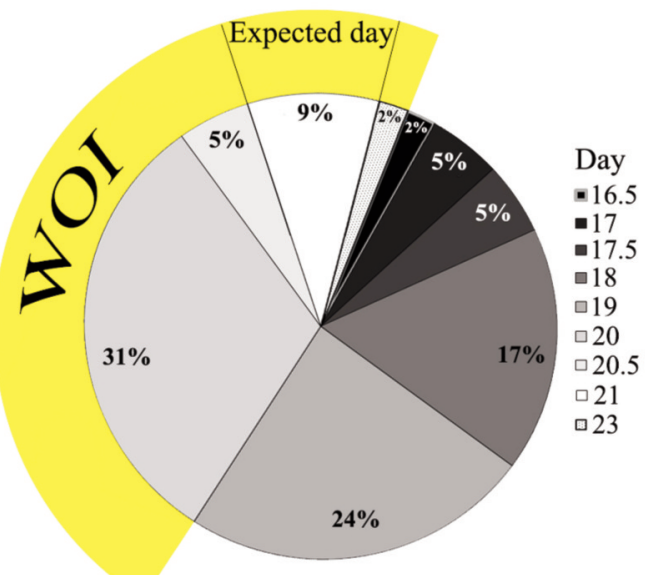

Biopsy at OV+7 (Natural cycle)

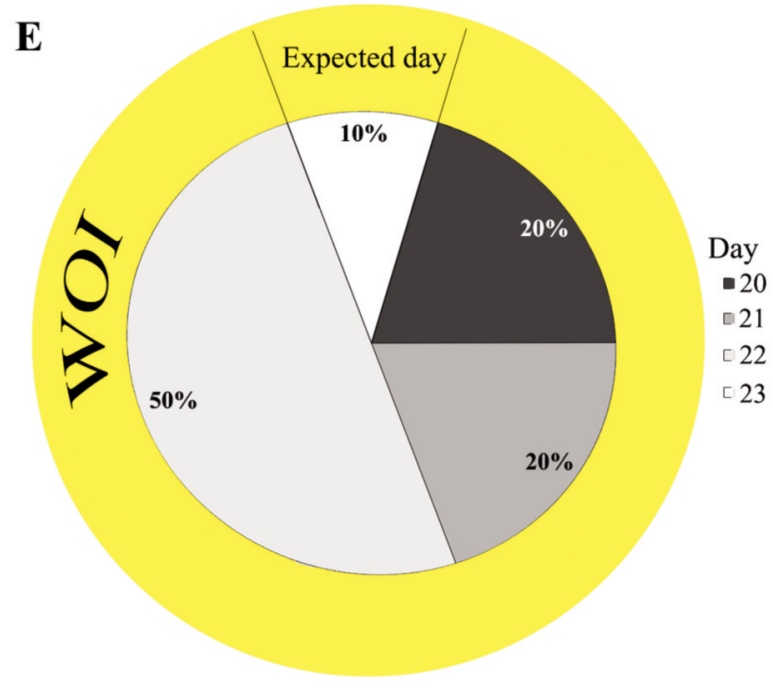

Biopsy at $\mathrm{OV}+9$ (Natural cycle)
B

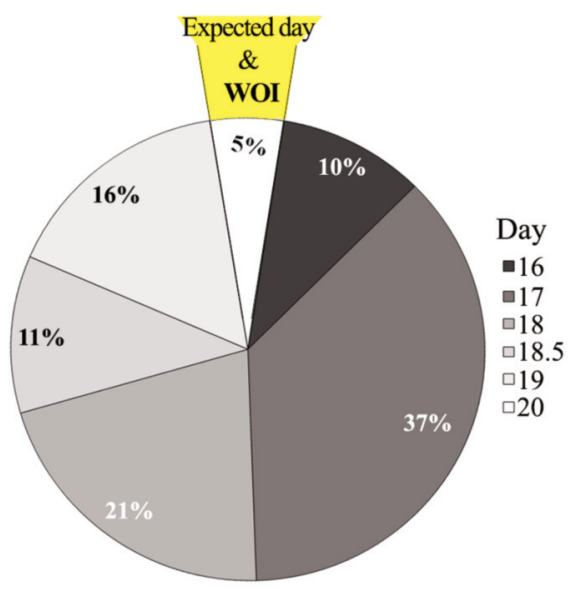

Biopsy at OV+6 (Natural cycle)

D

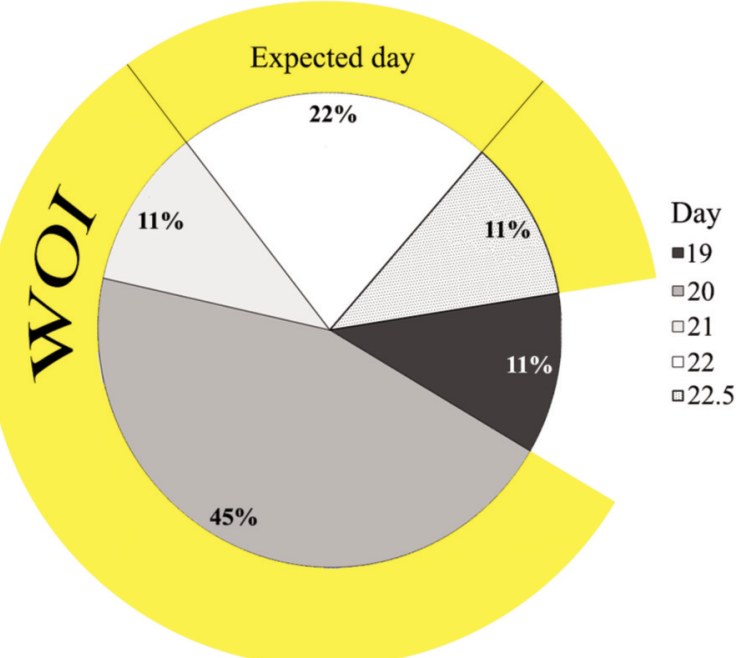

$\mathbf{F}$

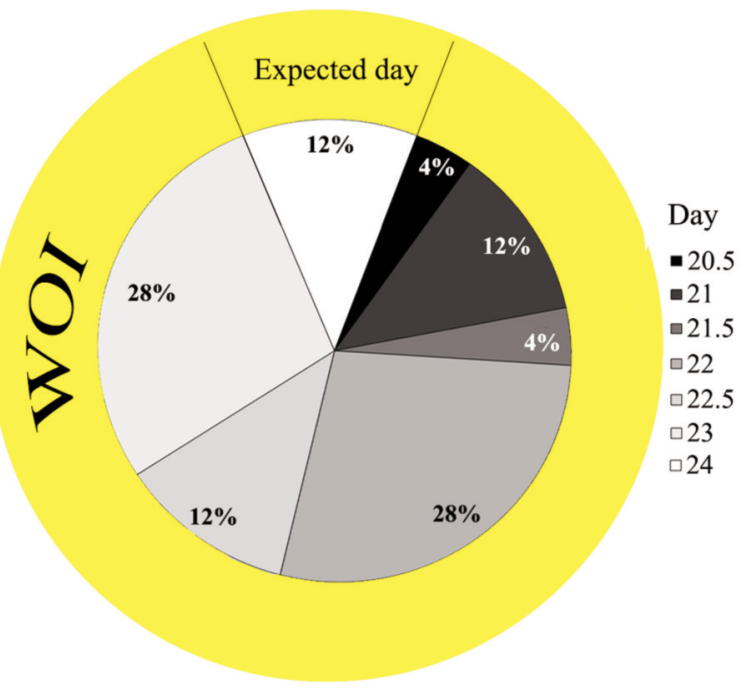

Biopsy at OV+10 (Natural cycle)

Figure 4. Endometrial dating according to the novel dating method during natural cycles. Dating was performed on biopsies from ovulation (OV) days $+5(A),+6(B),+7(C),+8(D),+9(E)$, and $+10(F)$. WOI: Window of implantation. 
A

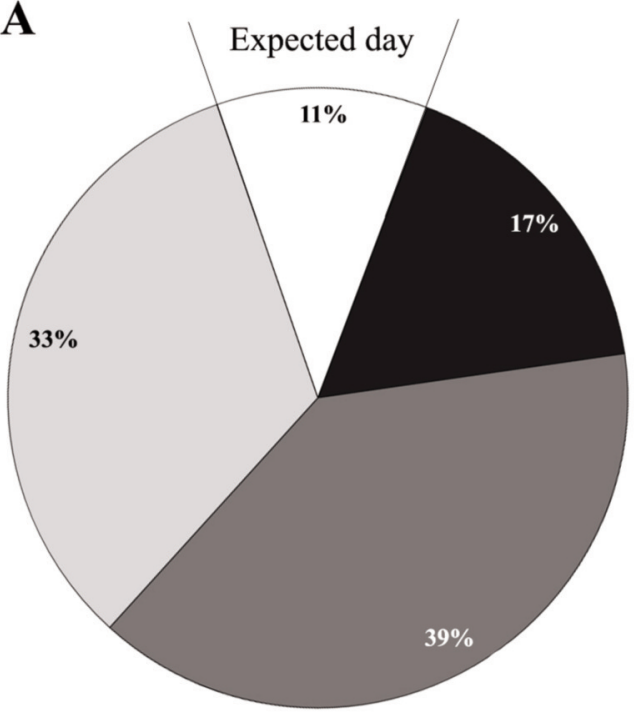

Biopsy at $\mathrm{P}+5$ (HRT cycle)
B

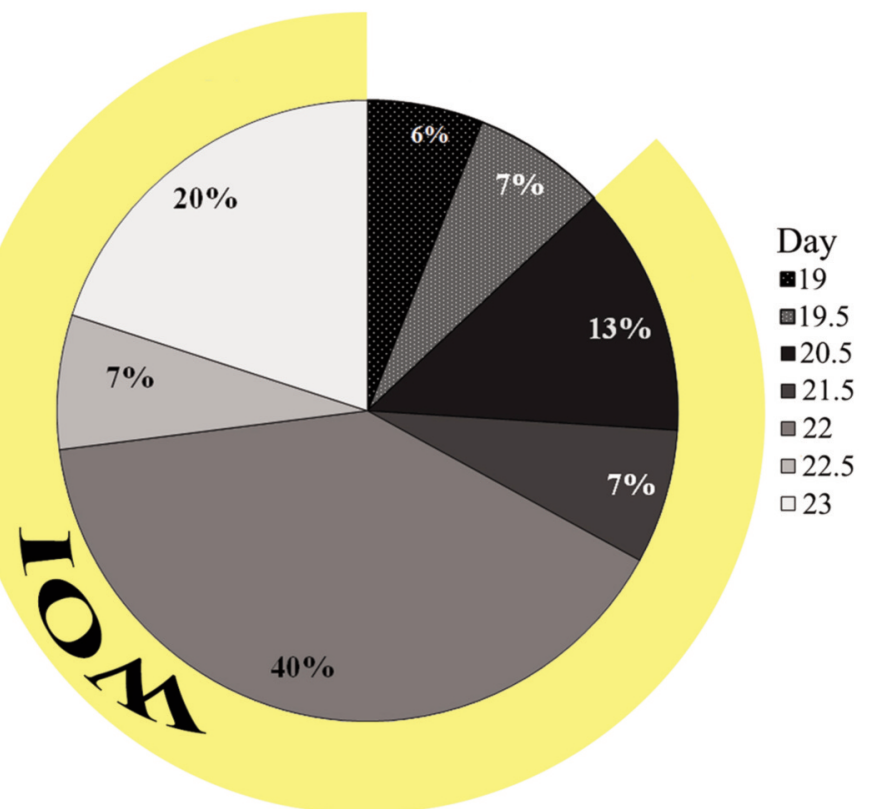

Biopsy at $\mathrm{P}+10$ (HRT cycle)

Figure 5. Endometrial dating according to the novel dating method on days $5(A)$ and $10(B)$ after progesterone $(P)$ in hormone replacement therapy (HRT) cycles. WOI: Window of implantation.

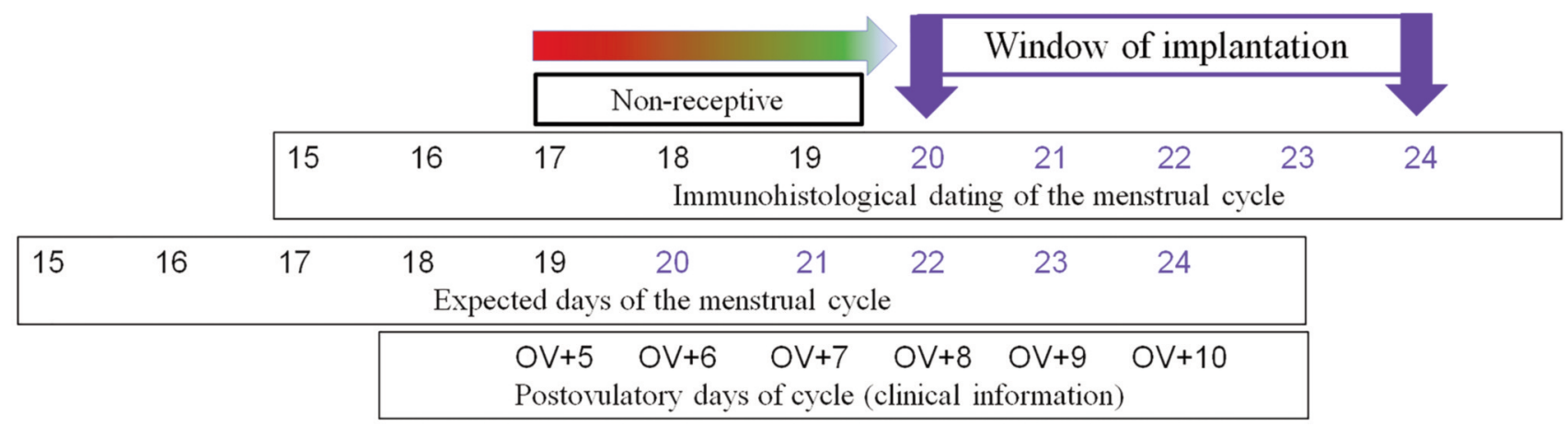

Figure 6. Comparison of immunohistological endometrial dating (novel method) with clinical days after ovulation (OV).

present modified endometrial dating method, it was also found that the duration of endometrial maturation differed individually among most of the participants, and endometrial maturation occurring $1.68 \pm 1.67$ days late was observed. This type of delay in endometrial maturation may lead to implantation failure, as the embryo and endometrium are not temporally coordinated. Previously, delayed endometrium was referred to as being "out of phase," but the present study observed individual endometrial maturation that appears to be normal and can nevertheless enter the window of implantation.
Since the present study included fertile women with normal menstruation (13), based on earlier investigations showing that endometrial maturation in subfertile women with normal menstruation is similar to that in fertile individuals (5), it can be postulated that the individual duration of endometrial maturation is not only restricted to subfertile patients but is also typical among fertile women, as demonstrated by Murray et al. (12). Several earlier studies have also often reported distinct variability in the length of the luteal phase as a normal finding even among fertile women, thus confirming the present results and the 
suggestion that the second half of the menstrual cycle is variable in both subfertile and fertile women $(10,11,19-22)$. The results of the present study show that morphological endometrial maturation and hormone receptor expression and down-regulation, in combination with the Ki-67 proliferation index, are similar in fertile (14) and subfertile patients. This was the reason why Noyes et al. decided to establish their dating method (which is currently the gold standard) on the basis of endometrium from infertile patients.

Van der Gaast et al. investigated 30 patients from a fertility clinic on day $\mathrm{OV}+5$ (16). After endometrial dating, in addition to immunohistochemical investigation of hormone receptors and proliferation marker Ki-67, they noted variation in endometrial maturation corresponding to days $\mathrm{OV}+2$ and +5.5 .

The results of the present study suggest that, particularly in the early secretory phase, endometrial maturation diverges from that under Noyes criteria. According to Noyes criteria, the implantation window starts on day $\mathrm{OV}+6$. However, using the new dating method showed that the implantation window usually started on day $\mathrm{OV}+8$, corresponding to histological day 20 in the present dating method. If a "delay" of 1.68 days is taken into account, most of the clinical days would match the maturation of the endometrium. Thus 'retarded' maturation is not a delay, but rather a normal variant, implying that in most fertile and subfertile women, the duration of the early luteal phase is 7 days rather than 5 days. However, endometrial maturation is an individual process that needs to be analyzed individually in each patient in clinical practice.

It has been suggested that delayed endometrial development results from a low progesterone level. However, Usadi et al. demonstrated that the level of circulating progesterone did not reflect the histological endometrial dating and so could not serve as a reliable bioassay for the quality of luteal function (23). The present results in the progesterone-treated group also confirmed that the delay in endometrial maturation is independent of the serum level of progesterone and can be seen in both HRT and natural cycles and is not a kind of corpus luteum insufficiency.

\section{Conclusion}

The findings of the present study show that the process of endometrial maturation is individually variable in subfertile women in both natural and HRT cycles. Endometrial maturation to reach histological day 20 , which is the start of the window of implantation, usually takes 7.7 days $(\mathrm{OV}+8)$, rather than 6 days $(\mathrm{OV}+6)$ based on Noyes criteria (5). Variation in the maturation process was also observed; maturation may be either accelerated or delayed relative to the expected maturation state at $\mathrm{OV}+8$. Many studies confirm the suspicion that these variations in endometrial maturation are normal in both fertile and subfertile women and thus do not represent any sort of unusual delay. These results suggest that each woman has her own individual maturation rate to reach the receptive window of implantation, and this stage should be matched with timing of embryo transfers.

\section{Funding}

No specific funding was received for the study. The research was supported financially by institutional funding from Erlangen University Hospital.

\section{Conflicts of Interest}

The Authors have no conflicts of interest to declare.

\section{Authors' Contributions}

J. Alfer: Conception and design, data acquisition, analysis and interpretation, drafting editing and final approval of the article; A. Fattahi: data analysis and interpretation, drafting, editing and final approval of the article; N. Bleisinger: data analysis and interpretation, drafting, editing and final approval of the article; J. Krieg: sample preparation, data acquisition, editing and final approval of the article; R. Behrens: sample preparation, data acquisition, editing and final approval of the article; R. Dittrich: conception and design, supervision; drafting, editing and final approval of the article; M.W. Beckmann: supervision and critical revisions, editing of the article, final approval; A. Hartmann: conception and design, supervision and critical revision, editing and final approval of the article; I. Classen-Linke: critical revision, editing and final approval of the article; R.M. Popovici: conception and design, sample preparation, supervision and data acquisition, editing and final approval of the article.

\section{Acknowledgements}

The Authors are grateful to all of the patients who participated in the study. They would also like to thank Dr. Michael Robertson for medical copy-editing.

\section{References}

1 Diaz-Gimeno P, Horcajadas JA, Martinez-Conejero JA, Esteban FJ, Alama P, Pellicer A and Simon C: A genomic diagnostic tool for human endometrial receptivity based on the transcriptomic signature. Fertil Steril 95(1): 50-60. e15, 2011. PMID: 20619403. DOI: $10.1016 /$ j.fertnstert.2010.04.063

2 Bassil R, Casper R, Samara N, Hsieh TB, Barzilay E, Orvieto R and Haas J: Does the endometrial receptivity array really provide personalized embryo transfer? J Assist Reprod Genet 35(7): 1301-1305, 2018. PMID: 29737471. DOI: 10.1007/s10815-018$1190-9$

3 Churchill S, Comstock I and Lathi R: Pregnancy outcomes after endometrial receptivity array in an infertile population. Fertil Steril 108(3): e360, 2017. DOI: 10.1016/j.fertnstert. 2017.07.1053 
4 Li TC, Rogers AW, Dockery P, Lenton EA and Cooke ID: A new method of histologic dating of human endometrium in the luteal phase. Fertil Steril 50(1): 52-60, 1988. PMID: 3289976. DOI: 10.1016/s0015-0282(16)60008-0

5 Noyes R, Hertig A and Rock J: Dating the endometrial biopsy. Am J Obstet Gynecol 122(2): 262-263, 1975. PMID: 1155504. DOI: $10.1016 /$ S0002-9378(16)33500-1

6 Scott RT, Snyder RR, Bagnall JW, Reed KD, Adair CF and Hensley SD: Evaluation of the impact of intraobserver variability on endometrial dating and the diagnosis of luteal phase defects. Fertil Steril 60(4): 652-657, 1993. PMID: 8405519. DOI: 10.1016/s0015-0282(16)56216-5

7 Driessen F, Holwerda PJ, vd Putte SC and Kremer J: The significance of dating an endometrial biopsy for the prognosis of the infertile couple. Int J Fertil 25(2): 112-116, 1980. PMID: 6117524.

8 Santoro N, Goldsmith LT, Heller D, Illsley N, McGovern P, Molina C, Peters S, Skurnick JH, Forst C and Weiss G: Luteal progesterone relates to histological endometrial maturation in fertile women. J Clin Endocrinol Metab 85(11): 4207-4211, 2000. PMID: 11095455. DOI: 10.1210/jcem.85.11.6974

9 Triwitayakorn A, Suwajanakorn S, Triratanachat S, Sampatanukul P, Pruksananonda K and Sereepapong W: Effects of initiation day of clomiphene citrate on the endometrium of women with regular menstrual cycles. Fertil Steril 78(1): 102-107, 2002. PMID: 12095498. DOI: $10.1016 / \mathrm{s} 0015-0282(02) 03192-8$

10 Lindhard A, Ravn V, Bentin-Ley U, Horn T, Bangsboell S, Rex S, Toft B and Soerensen S: Ultrasound characteristics and histological dating of the endometrium in a natural cycle in infertile women compared with fertile controls. Fertil Steril 86(5): 1344-1355, 2006. PMID: 16978615. DOI: 10.1016/ j.fertnstert.2006.03.052

11 Coutifaris C, Myers ER, Guzick DS, Diamond MP, Carson SA, Legro RS, McGovern PG, Schlaff WD, Carr BR, Steinkampf MP, Silva S, Vogel DL, Leppert PC and Network NNCRM: Histological dating of timed endometrial biopsy tissue is not related to fertility status. Fertil Steril 82(5): 1264-1272, 2004. PMID: 15533340. DOI: 10.1016/j.fertnstert.2004.03.069

12 Murray MJ, Meyer WR, Zaino RJ, Lessey BA, Novotny DB, Ireland $\mathrm{K}$, Zeng D and Fritz MA: A critical analysis of the accuracy, reproducibility, and clinical utility of histologic endometrial dating in fertile women. Fertil Steril 81(5): 1333-1343, 2004. PMID: 15136099. DOI: 10.1016/j.fertnstert.2003.11.030

13 Classen-Linke I, Alfer J, Hey S, Krusche CA, Kusche M and Beier HM: Marker molecules of human endometrial differentiation can be hormonally regulated under in-vitro conditions as in-vivo. Hum Reprod Update 4(5): 539-549, 1998. PMID: 10027607. DOI: 10.1093/humupd/4.5.539
14 Cutting R, Morroll D, Roberts SA, Pickering S, Rutherford A, Bfs and Ace: Elective single embryo transfer: Guidelines for practice British Fertility Society and Association of Clinical Embryologists. Hum Fertil 11(3): 131-146, 2008. PMID: 18766953. DOI: $10.1080 / 14647270802302629$

15 Kaufmann C: Therapeutics with hormones of the ovary: (Section of Therapeutics and Pharmacology). Proc R Soc Med 27: 849863, 1934. PMID: 19989793.

16 van der Gaast MH, Macklon NS, Beier-Hellwig K, Krusche CA, Fauser BC, Beier HM and Classen-Linke I: The feasibility of a less invasive method to assess endometrial maturationcomparison of simultaneously obtained uterine secretion and tissue biopsy. BJOG 116(2): 304-312, 2009. PMID: 19076963. DOI: $10.1111 / \mathrm{j} .1471-0528.2008 .02039 . \mathrm{x}$

17 Usadi RS, Murray MJ, Bagnell RC, Fritz MA, Kowalik AI, Meyer WR and Lessey BA: Temporal and morphologic characteristics of pinopod expression across the secretory phase of the endometrial cycle in normally cycling women with proven fertility. Fertil Steril 79(4): 970-974, 2003. PMID: 12749439. DOI: $10.1016 / \mathrm{s} 0015-0282(02) 04929-4$

18 Rock J and Bartlett MK: Biopsy studies of human endometrium: Criteria of dating and information about amenorrhea, menorrhagia, and time of ovulation. J Am Med Assoc 108(24): 2022-2028, 1937. PMID: 12255649. DOI: 10.1001/jama. 1937.02780240014006

19 Johannisson E, Parker RA, Landgren BM and Diczfalusy E: Morphometric analysis of the human endometrium in relation to peripheral hormone levels. Fertil Steril 38(5): 564-571, 1982. PMID: 7128842. DOI: 10.1016/s0015-0282(16)46636-7

20 Lenton EA, LANDGREN BM and Sexton L: Normal variation in the length of the luteal phase of the menstrual cycle: Identification of the short luteal phase. BJOG 91(7): 685-689, 1984. PMID: 6743610. DOI: 10.1111/j.1471-0528.1984.tb04831.x

21 Vollman RF: The menstrual cycle. Major Probl Obstet Gynecol 7: 1-193, 1977. PMID: 836520.

22 Münster K, Schmidt L and Helm P: Length and variation in the menstrual cycle-a cross-sectional study from a danish county. BJOG 99(5): 422-429, 1992. PMID: 1622917. DOI: 10.1111/ j.1471-0528.1992.tb13762.x

23 Usadi RS, Groll JM, Lessey BA, Lininger RA, Zaino RJ, Fritz MA and Young SL: Endometrial development and function in experimentally induced luteal phase deficiency. J Clin Endocrinol Metab 93(10): 4058-4064, 2008. PMID: 18647810. DOI: $10.1210 /$ jc.2008-0460

Received April 5, 2020

Revised April 16, 2020

Accepted April 27, 2020 